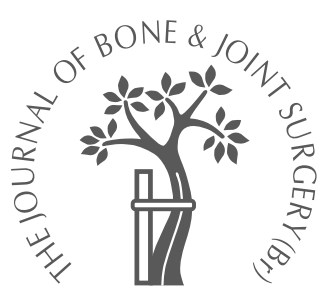

T. Aihara,

K. Takahashi,

A. Ogasawara,

E. Itadera,

Y. Ono,

H. Moriya

From Chousei

Hospital, Mobara

City, Japan

\title{
Intervertebral disc degeneration associated with lumbosacral transitional vertebrae
}

\author{
A CLINICAL AND ANATOMICAL STUDY
}

T. Aihara, MD, Orthopaedic Surgeon

A. Ogasawara, MD,

Orthopaedic Surgeon

E. Itadera, MD,

Orthopaedic Surgeon

Y. Ono, MD, Head of

Hospital

Department of Orthopaedic

Surgery

Chousei Hospital, 2777

Honnou, Mobara-City, Chiba

299-4114, Japan.

K. Takahashi, MD,

Associate Professor

- H. Moriya, MD, Professor

and Head of Department

Department of Orthopaedic

Surgery (J1)

Graduate School of

Medicine, Chiba University,

1-8-1 Inohana, Chiba-City,

Chiba 260-8677, Japan.

Correspondence should be sent to $\mathrm{Dr}$ T. Aihara at

Funabashi Orthopedic

Hospital, 1-833 Hazama,

Funabashi-City, Chiba 274-

0822, Japan; e-mail:

aiharaty@h3.dion.ne.jp

(C)2005 British Editorial

Society of Bone and

Joint Surgery

doi:10.1302/0301-620X.87B5.

$15727 \$ 2.00$

$J$ Bone Joint Surg [Br]

2005;87-B:687-91.

Received 15 June 2004;

Accepted after revision

18 January 2005

We studied 52 patients, each with a lumbosacral transitional vertebra. Using MRI we found that the lumbar discs immediately above the transitional vertebra were significantly more degenerative and those between the transitional vertebrae and the sacrum were significantly less degenerative compared with discs at other levels. We also performed an anatomical study using $\mathbf{7 0}$ cadavers. We found that the iliolumbar ligament at the level immediately above the transitional vertebra was thinner and weaker than it was in cadavers without a lumbosacral transitional vertebra.

Instability of the vertebral segment above the transitional vertebra because of a weak iliolumbar ligament could lead to subsequent disc degeneration which may occur earlier than at other disc levels. Some stability between the transitional vertebra and the sacrum could be preserved by the formation of either an articulation or by bony union between the vertebra and the sacrum through its transverse process. This may protect the disc from further degeneration in the long term.

The iliolumbar ligament connects the transverse process of the fifth lumbar vertebra to the ilium, stabilising onto the pelvis (Fig. 1). There have been a number of reports concerning its anatomy and biomechanical functions, ${ }^{1-9}$ but only a few on its clinical significance because of the difficulty in accurately determining biomechanical strength and size in vivo. ${ }^{10-12}$ Aihara et $\mathrm{al}^{12}$ performed an anatomical study of the iliolumbar ligament in association with degeneration of the lumbosacral disc. They concluded that if the iliolumbar ligament in men was longer and had a smaller cross-sectional area, the lumbosacral junction could not be adequately stabilised by the ligament, resulting in advanced L5-S1 disc degeneration. They also concluded that the cross-sectional area of the iliolumbar ligament may be related to degeneration of the lumbosacral disc in women. However, in that study, cadavers with a lumbosacral transitional vertebra were not included.

Elster ${ }^{13}$ suggested that movement between the transitional vertebra and the sacrum was very limited, whereas the disc space above the transitional vertebra was more mobile. He proposed that hypermobility at the interspace above the transitional vertebra resulted in an increased risk of subsequent disc degeneration. However, he could not identify any studies in the literature on the biomechanics of transi- tional vertebrae. We have suggested that hypermobility at the vertebral segments above the transition, with the subsequent risk of disc degeneration, is due to the biomechanical strength of the iliolumbar ligament at the level immediately above the transitional vertebra.

To the best of our knowledge, there have been no studies which have demonstrated the morphology of the iliolumbar ligament in association with the lumbosacral transitional vertebra. In addition, there have been only two previous reports in the literature concerning degeneration of the intervertebral disc in association with lumbosacral transitional vertebrae, based on MRI. ${ }^{14,15}$ In this study we have attempted to determine the influence of the lumbosacral transitional vertebra on disc degeneration and establish whether the morphology of the iliolumbar ligament is involved in degeneration associated with a lumbosacral transitional vertebra.

\section{Patients and Methods}

Clinical study. We examined the lumbar spines of 66 patients with a type II, type III or type IV lumbosacral transitional vertebra according to Castellvi, Goldstein and Chan, ${ }^{16}$ using plain radiography and MRI. All the patients had low back pain with or without sciatica. From this group, we excluded ten patients with L4 degenerative spondylolisthesis, two with L4 


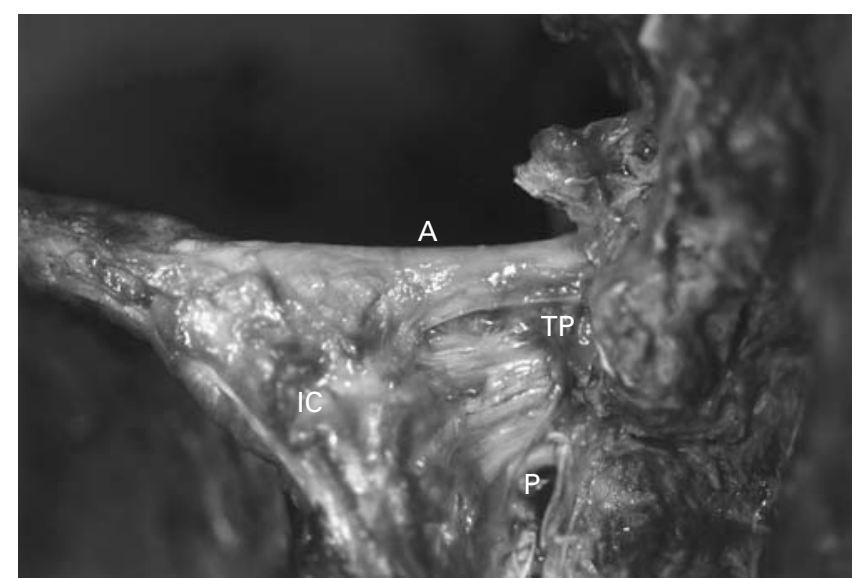

Fig. 1

Photograph of a specimen from an 82-year-old female showing that the iliolumbar ligament has two separate bands. The anterior band $(A)$ inserts into the anterior margin of the iliac crest (IC) and the posterior band (P) into the posterior margin of the crest ${ }^{2,3,9,10,12}$ (TP, L5 transverse process).

spondylolytic spondylolisthesis, one who had undergone previous lumbar spinal surgery, and one with L3 degenerative spondylolisthesis. As stated by Vergauwen et al, ${ }^{17}$ the entire spine is not always visualised and so identification of the fifth lumbar vertebra is not always possible. Therefore, the vertebra immediately above the transitional vertebra was termed L4. Our remaining 52 patients consisted of 27 men with a mean age of 53.4 years $(13$ to 86$)$ and 25 women with a mean age of 47.8 years ( 16 to 80 ). The ages of the men and women were compared using an unpaired $t$ test, but there was no significant difference $(\mathrm{p}=0.28)$.

Using plain radiography, the lumbosacral transitional vertebrae were classified according to Castellvi et al. ${ }^{16}$ In type I there is a large transverse process, in type II there is a diarthrodial joint between the transverse process and the sacrum (a, unilateral; b, bilateral), in type III a true bony union between the transverse process and the sacrum ( $a$, unilateral; b, bilateral), and in type IV there is type II on one side and type III on the other. Castellvi et $\mathrm{al}^{16}$ reported that there were no clinical differences between patients with a type-I transitional vertebra and the normal population. As described by Wigh and Anthony ${ }^{18}$ when the lowest lumbar transverse process was simply enlarged, this is not a sign of the vertebra being transitional. Therefore, in our study all patients with a type-I transitional vertebra were excluded. ${ }^{19}$

Using T2-weighted sagittal MRI, the grade of L2/3, L3/4, L4-transitional vertebra (TV) and TV-S1 disc degeneration was determined according to the criteria described by Thompson. ${ }^{11,20}$ The T2-weighted sagittal images (repeat time/echo time [TR/TE] 3000/100 ms) were obtained with a 0.5 T MRI unit (MRVectra; GE Yokogawa Medical Systems, Tokyo, Japan). The grade of disc degeneration was determined by assessing the degenerative status of the nucleus pulposus, annulus fibrosus, the vertebral end-plate and the vertebral body. Grade I indicated normal and grade $\mathrm{V}$ the most advanced degeneration. ${ }^{20}$ In our study, we scored degeneration using a scale of 1 to 5 , based on grades I to $\mathrm{V}$ according to Thompson's criteria. ${ }^{21,22}$

We compared the grades of disc degeneration using Scheffe's F test for differences in L2/3, L3/4, L4-TV and TVS1 levels. Miller et $\mathrm{al}^{21}$ reported that male lumbar discs were significantly more degenerative than female lumbar discs for most age groups. They suggested that higher mechanical stress on the male intervertebral disc may contribute to its earlier and more severe degeneration. Therefore, men and women were evaluated separately in our study. Since the grade of lumbosacral disc degeneration has been shown to be influenced by age, ${ }^{23,24}$ we also separately analysed the younger 14 men with a mean age of 38.6 years (13 to 54), the older 13 men with a mean age of 69.4 years (59 to 86), the younger 12 women with a mean age of 31.3 years (16 to 50$)$ and the older 13 women with a mean age of 63.0 years ( 51 to 80$)$. A p value of less than 0.05 was considered to be statistically significant.

Anatomical study. We dissected 78 cadavers and performed anteroposterior and lateral radiography of the lumbosacral spine. From this group we excluded four cadavers with ossification of the iliolumbar ligament and four with an L5 spondylolisthesis. Of the remaining 70 cadavers, eight had a lumbosacral transitional vertebra (types II to IV of Castellvi et $\left.\mathrm{al}^{16}\right)$. Of these, four were men and four were women, with a mean age at death of 75.1 years (63 to 90). Of the 62 cadavers without a lumbosacral transitional vertebrae, 30 were men and 32 were women with a mean age at death of 73.0 years (39 to 102). There was no significant difference in age between the cadavers with a lumbosacral transitional vertebra and those without (unpaired $t$-test, $\mathrm{p}=$ $0.65)$.

We compared the morphology of the iliolumbar ligament in the eight cadavers with a lumbosacral transitional vertebra and the 62 cadavers without.

\section{Results}

Clinical study. A type-IIa transitional vertebra occurred in ten men and ten women, type IIb in one man and three women, type IIIa in four men and four women, type IIIb in 11 men and five women and type IV in one man and three women.

The disc degeneration and mean degeneration scores at each level in men and women are shown in Table I. The discs immediately above the L4-TV were significantly degenerative compared with those at other levels. However, there was no significant difference between L2/3 and L3/4 levels in terms of disc degeneration (men: $\mathrm{p}=0.85$; women: $\mathrm{p}=0.96)$. The discs between the transitional vertebra and the sacrum TV-S1 were significantly less degenerative than those at other levels. Only two discs in men and three in women were not degenerative immediately above the L4TV. However, $93 \%$ of the discs in men and $88 \%$ of the discs in women were degenerative immediately above the transi- 
Table I. Disc degeneration and mean degeneration scores at L2/3, L3/4, L4-TV* and TV-S1 levels in 27 men and 25 women

\begin{tabular}{|c|c|c|c|c|c|c|}
\hline \multirow[b]{2}{*}{ Level } & \multicolumn{5}{|c|}{ Disc degeneration scores } & \multirow{2}{*}{$\begin{array}{l}\text { Mean degeneration } \\
\text { score } \pm \text { SD }\end{array}$} \\
\hline & 1 & 2 & 3 & 4 & 5 & \\
\hline \multicolumn{7}{|l|}{ Men } \\
\hline $\mathrm{L} 2 / 3$ & 6 & 10 & 8 & 2 & 1 & $2.33 \pm 1.0^{\dagger}$ \\
\hline $\mathrm{L} 3 / 4$ & 4 & 9 & 10 & 3 & 1 & $2.56 \pm 1.0^{\dagger}$ \\
\hline L4-TV & 2 & 0 & 11 & 13 & 1 & $3.41 \pm 0.9^{\dagger}$ \\
\hline TV-S1 & 24 & 2 & 0 & 1 & 0 & $1.19 \pm 0.6^{\dagger}$ \\
\hline Total & 36 & 21 & 29 & 19 & 3 & \\
\hline \multicolumn{7}{|l|}{ Women } \\
\hline $\mathrm{L} 2 / 3$ & 7 & 9 & 6 & 3 & 0 & $2.20 \pm 1.0^{\ddagger}$ \\
\hline $\mathrm{L} 3 / 4$ & 8 & 4 & 9 & 4 & 0 & $2.36 \pm 1.1^{\ddagger}$ \\
\hline L4-TV & 3 & 1 & 9 & 8 & 4 & $3.36 \pm 1.2^{\ddagger}$ \\
\hline TV-S1 & 19 & 4 & 2 & 0 & 0 & $1.32 \pm 0.6^{\ddagger}$ \\
\hline Total & 37 & 18 & 26 & 15 & 4 & \\
\hline
\end{tabular}

* TV, transitional vertebrae

$\dagger$ significant difference between levels L2/3 and L4-TV $(p=0.0005)$ levels L3/4 and L4-TV ( $p=0.0099)$, levels L4-TV and TV-S1 $(p<0.0001)$ levels $\mathrm{L} 2 / 3$ and TV-S1 $(p=0.0002)$ and between levels L3/4 and TV-S1 $(\mathrm{p}<0.0001)$

\# significant difference between levels L2/3 and L4-TV $(p=0.0015)$, levels L3/4 and L4-TV ( $p=0.0085)$, levels L4-TV and TV-S1 $(p<0.0001)$, levels L2/3 and TV-S1 $(p=0.027)$ and between levels L3/4 and TV-S1 $(p=0.0056)$

Table II. Mean degeneration scores at L2/3, L3/4, L4-TV* and TV-S1 levels in younger and older men and women

\begin{tabular}{clllllll}
\hline & \multicolumn{2}{l}{ Younger } & & & \multicolumn{2}{l}{ Older } \\
\cline { 2 - 3 } Level & Number & Mean & SD & & Number & Mean & SD \\
\hline Men & 14 & & & & 13 & & \\
L2/3 & & $1.71^{\dagger}$ & 0.7 & & $3.00^{\ddagger}$ & 0.9 \\
L3/4 & & $2.00^{\dagger}$ & 0.8 & & $3.15^{\ddagger}$ & 0.9 \\
L4-TV & & $3.07^{\dagger}$ & 1.0 & & $3.77^{\ddagger}$ & 0.6 \\
TV-S1 & & $1.00^{\dagger}$ & 0 & & $1.39^{\ddagger}$ & 0.9 \\
Women & 12 & & & & & & \\
L2/3 & & $1.50^{\dagger}$ & 0.5 & & $2.85^{\ddagger}$ & 0.9 \\
L3/4 & & $1.67^{\dagger}$ & 1.0 & & $3.00^{\ddagger}$ & 0.8 \\
L4-TV & & $2.92^{\dagger}$ & 1.3 & & $3.77^{\ddagger}$ & 0.9 \\
TV-S1 & & $1.17^{\dagger}$ & 0.6 & & $1.46^{\ddagger}$ & 0.7 \\
\hline
\end{tabular}

* TV, transitional vertebrae

$\dagger$ significant difference between levels L2/3 and L4-TV $(p<0.006)$, levels L3/4 and L4-TV ( $p<0.02)$, levels L4-TV and TV-S1 $(p<0.005)$, and between levels L3/4 and TV-S1 (only in men, $p=0.0081$ )

\# significant difference between levels L2/3 and TV-S1 ( $p<0.002$ ) levels L3/4 and TV-S1 ( $<0.005)$, and between levels L4-TV and TV-S1 $(p<0.0001)$

Table III. The morphology of the iliolumbar ligaments at the level immediately above the transitional vertebra

\begin{tabular}{|c|c|c|c|c|c|c|}
\hline \multirow{3}{*}{$\begin{array}{l}\text { Age } \\
\text { (yrs) }\end{array}$} & \multirow[b]{3}{*}{ Gender } & \multirow{3}{*}{$\begin{array}{l}\text { Type of } \\
\text { transitional } \\
\text { vertebra }^{\dagger}\end{array}$} & \multirow{2}{*}{\multicolumn{2}{|c|}{$\begin{array}{l}\text { Bands of iliolumbar } \\
\text { ligaments* } \\
\text { Right }\end{array}$}} & \multirow{2}{*}{\multicolumn{2}{|c|}{ Left }} \\
\hline & & & & & & \\
\hline & & & Anterior & Posterior & Anterior & Posterior \\
\hline 63 & $\mathrm{M}$ & Illa & $\mathrm{M}$ & $\mathrm{F}$ & $\mathrm{M}$ & $F$ \\
\hline 66 & $\mathrm{M}$ & IV & $\mathrm{L}$ & $\mathrm{F}$ & $L$ & $\mathrm{~F}$ \\
\hline 74 & $\mathrm{M}$ & Ila & $L$ & $\mathrm{M}$ & $\mathrm{F}$ & $\mathrm{M}$ \\
\hline 83 & $\mathrm{M}$ & Ila & $L$ & $\mathrm{~F}$ & $L$ & $L$ \\
\hline 68 & $\mathrm{~F}$ & Illa & $\mathrm{F}$ & $\mathrm{F}$ & $\mathrm{F}$ & $\mathrm{F}$ \\
\hline 68 & $\mathrm{~F}$ & IIIb & $L$ & $\mathrm{~F}$ & $\mathrm{~L}$ & $\mathrm{~F}$ \\
\hline 89 & $\mathrm{~F}$ & Ila & $\mathrm{M}$ & $\mathrm{F}$ & $\mathrm{F}$ & $\mathrm{L}$ \\
\hline 90 & $\mathrm{~F}$ & Illb & $\mathrm{F}$ & $M$ & $\mathrm{~F}$ & $\mathrm{~F}$ \\
\hline
\end{tabular}

* L, ligament; $F$, fascia; $M$, missing

+ Castellvi et al $^{16}$

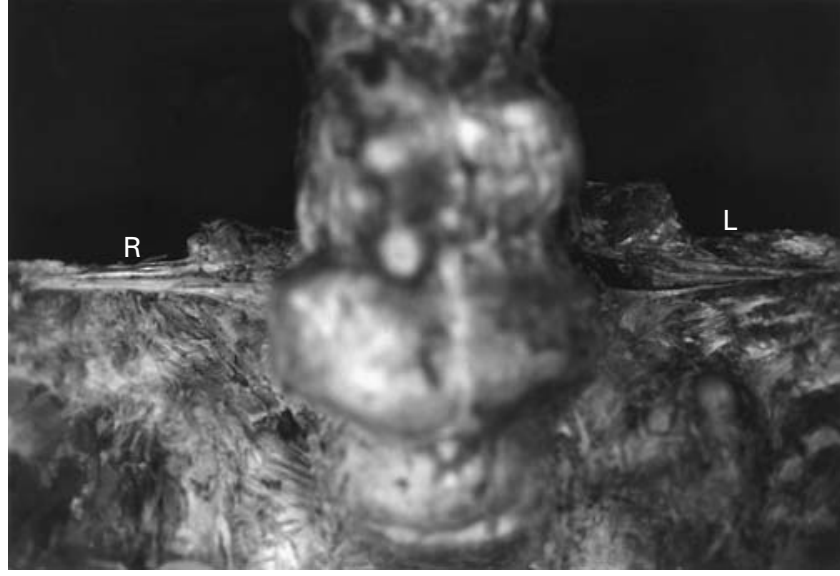

Fig. 2

Photograph of a 74-year-old male specimen with a lumbosacral transitional vertebra (Castellvi type Ila). On the right $(R)$ the anterior band of the iliolumbar ligament immediately above the transitional vertebra is much thinner than the normal L5 branch; the posterior band is missing. That on the left $(L)$ at the same level seems to be fascia rather than a ligament. The posterior band is missing.

tional vertebra. Discs in three men and two women were slightly vestigial between the transitional vertebra and the sacrum (TV-S1), but they were not devoid of nuclear material or degenerative. Only two discs in men and four in women were mildly degenerative between the transitional vertebra and the sacrum (TV-S1). However, $89 \%$ of the discs in men and $76 \%$ in women were not degenerative between the transitional vertebra and the sacrum (Table I).

The mean degeneration scores at each level in the 14 younger and the 13 older men are shown in Table II. In the younger group, the discs immediately above the L4-TV were significantly degenerative compared with those at other levels. The L3/4 discs were also significantly degenerative compared with the discs between the transitional vertebra and the sacrum in the 14 younger men. In the 13 older men, the discs between the transitional vertebra and the sacrum S1 were significantly less degenerative compared with those at other levels (Table II).

The mean degeneration scores at each level in the 12 younger and 13 older women are shown in Table II. In the 12 younger women, the discs immediately above the L4-TV were significantly degenerative compared with those at other levels. In the 13 older women, the discs between the transitional vertebra and the sacrum TV-S1 were significantly less degenerative compared with those at other levels (Table II).

Anatomical study. The morphology of the iliolumbar ligament at the level immediately above the transitional vertebra is shown in Table III. At this level it was much thinner and weaker than the L5 branches in cadavers without a lumbosacral transitional vertebra (Fig. 2). Although it was difficult to differentiate between anterior and posterior bands of the iliolumbar ligament in several specimens, six 
of the anterior and 11 of the posterior bands at this level had the appearance of fascia rather than ligament. Furthermore, three of the anterior and posterior bands of the iliolumbar ligament were missing at the level immediately above the transitional vertebra. The iliolumbar ligament at the transitional vertebra consisted of dense fibrous tissue firmly connected to the ilium without an identifiable ligament. In the case of a type-IIa or type-IIIa transitional vertebrae, the transverse processes of the normal side (opposite side to the diarthrodial joint or the bony union) showed a thick, strong iliolumbar ligament extending to the ilium. The L4 branches of the iliolumbar ligament of the cadavers without a lumbosacral transitional vertebra were preserved in several of the specimens and were much thinner and weaker than the L5 branches.

\section{Discussion}

It is difficult to visualise the iliolumbar ligament in vivo by imaging techniques because of the complexity and variability of the musculotendinous, fascial and ligamentous structures present in the lumbosacral region. ${ }^{10,12,25}$ Basadonna, Gasparini and Rucco ${ }^{26}$ reported an analysis by MRI of the anatomical characteristics of the insertion of the iliolumbar ligament in men. However, major problems remain in determining the biomechanical strength of the iliolumbar ligament and accurately delineating its morphology in vivo. Therefore, we compared the morphology of the ligament in the eight cadavers with a lumbosacral transitional vertebra with that in the 62 cadavers without.

Previous studies have reported that injuries, extreme loading, and rotational stress are important aetiological factors in disc degeneration. ${ }^{27}$ Farfan et $\mathrm{al}^{28}$ and Farfan ${ }^{29}$ have suggested that torsional load is the mechanical basis for disc degeneration. They demonstrated how the iliolumbar ligament provides torsional stability and therefore protects the lumbosacral disc from degeneration, especially in the presence of facet pathology. ${ }^{25}$ Aihara et al ${ }^{12}$ reported that if the iliolumbar ligament in men was short and had a large cross-sectional area (especially the posterior band of the ligament), the lumbosacral junction could be stabilised by the ligaments thereby avoiding L5-S1 disc degeneration. However, the L4/5 disc may be prone to degeneration and the cross-sectional area of the iliolumbar ligament, especially the posterior band, may have a role in lumbosacral disc degeneration in women. Miller et $\mathrm{al}^{21}$ reported that the L3/4 and the L4/5 discs were significantly degenerative compared with the L2/3 disc, although there were no significant differences in the grade of degeneration between the L5-S1 disc and the L3/4 or L4/5 discs. We suspect that this is because the iliolumbar ligament protects the L5-S1 disc from degeneration.

Keim stated that lumbosacral transitional vertebrae produced abnormal torque moments at the vertebral segments above the transition, and that this could eventually result in disc degeneration. ${ }^{16}$ We think that hypermobility and abnormal torque moments at the vertebral segments above the transition, which subsequently can lead to disc degeneration are because the iliolumbar ligament at that level is thinner and weaker, especially the posterior band, than the normal L5 branches. We also think that movement between the transitional vertebra and the sacrum is probably very limited owing to articulation or bony union of this vertebra with the sacrum through its transverse process and also to the iliolumbar ligaments at the transitional vertebra, dense fibrous tissue or thick strong ligaments firmly connecting to the ilium. Therefore, the discs between the transitional vertebra and the sacrum were not degenerative. It is difficult to know whether the iliolumbar ligaments were thin and weak before disc degeneration occurred or whether this was secondary to the degeneration. To our knowledge, this has not been previously reported. We suspect that the disc degeneration above the transitional vertebra is created by a congenitally thin and weak iliolumbar ligament.

Luoma et $\mathrm{al}^{14}$ reported that the transitional vertebra was associated with an increased risk of degenerative changes in the disc above in young men (18 to 20 years) and with a decreased risk in the disc below in middle-aged men (40 to 45 years). In our study, the discs immediately above the transitional vertebra were significantly degenerative compared with those at other levels in younger men (mean age of 38.6 years) and women (mean age of 31.3 years). The L3/4 discs were significantly degenerative compared with those between the transitional vertebra and the sacrum in the younger men. There was no significant difference between the ages of the younger men and women. The grade of lumbosacral disc degeneration is thought to be influenced by age. ${ }^{23,24}$ Miller et $\mathrm{al}^{21}$ reported that male lumbar discs were significantly more degenerative than female discs across most age groups, and suggested that higher mechanical stress on the male intervertebral disc may contribute to its earlier and more severe degeneration. We suspect that in younger men, the L4-TV discs were already degenerative, the L3/4 discs were also degenerative, but L2/3 discs were not yet so degenerative and TV-S1 discs were not yet degenerative at all. In the younger women, the L4-TV discs were already degenerative, while L2/3, L3/4 and TV-S1 discs were not yet so degenerative.

In our study, the discs between the transitional vertebra and the sacrum were significantly less degenerative compared with those at other levels in the older men and women. We consider that in these groups the L4-TV discs were already degenerative and the L3/4 and L2/3 discs were also degenerative but the TV-S1 discs were as yet not very degenerative. It is generally thought that man has problems in the lower lumbar spine because of his upright stance and associated axial loading together with the shear forces which are greatest in the lower lumbar region. Hypermobility and altered stresses become concentrated at the level immediately above the transitional vertebra. ${ }^{17}$ We consider that the hypermobility and abnormal torque moments at the level immediately above the transitional vertebra are more severe than at the other levels. This exposes the discs 
immediately above the transitional vertebra to the potential of degeneration earlier than at other disc levels. It is likely that movement between the transitional vertebra and the sacrum is very restricted, and therefore the disc at this level does not become so degenerative with ageing.

In vivo, a number of anatomical and other factors may influence lumbosacral disc degeneration. ${ }^{21,23,24,27}$ Battie et $\mathrm{al}^{24}$ found unexplained variability in the lower lumbar region. Our study demonstrated that instability of the vertebral segments above the transitional vertebra because of the weak iliolumbar ligaments could subsequently lead to disc degeneration sooner than in the discs at other levels. The stability between the transitional vertebra and the sacrum arises from the formation of either an articulation or by bony union of this vertebra with the sacrum through its transverse process. Also the iliolumbar ligaments at the transitional vertebra consist of strong, dense fibrous tissue which may offer further protection against disc degeneration.

\section{Supplementary material}

藏 A further opinion by Mr Michael Edgar is available with the electronic version of this article on our website at www.jbjs.org.uk

We wish to thank former Professor Y. Shimada of the Department of Anatomy, Graduate School of Medicine, Chiba University, and Dr. K. Matsuki for their help and critical comments.

No benefits in any form have been received from a commercial party related directly or indirectly to the subject of this article.

\section{References}

1. Shellshear JL, Macintosh NWG. The transverse process of the fifth lumbar vertebra. In: Shellshear JL, Macintosh NWG, eds. Surveys of anatomical fields. Sydney: Grahame, 1949:21-32.

2. Leong JCY, Luk KDK, Chow DHK, Woo CW. The biomechanical functions of the iliolumbar ligament in maintaining stability of the lumbosacral junction. Spine 1987; 12:669-74.

3. Luk KDK, Ho HC, Leong JCY. The iliolumbar ligament: a study of its anatomy, development and clinical significance. J Bone Joint Surg [Br] 1986;68-B:197-200.

4. Hanson P, Sonesson B. The anatomy of the iliolumbar ligament. Arch Phys Med Rehabil 1994;75:1245-6.

5. Fujiwara A, Tamai K, Yoshida $\mathbf{H}$, et al. Anatomy of the iliolumbar ligament. Clin Orthop 2000:380:167-72.

6. Grobler LJ, Novotny JE, Wilder DG, Frymoyer JW, Pope MH. L4-5 isthmic spondylolisthesis: a biomechanical analysis comparing stability in L4-5 and L5-S isthmic spondylolisthesis. Spine 1994;19:222-7.
7. Chow DHK, Luk KDK, Leong JCY, Woo CW. Torsional stability of the lumbosacral junction: significance of the iliolumbar ligament. Spine 1989;14:611-15.

8. Yamamoto I, Panjabi MM, Oxland TR, Crisco JJ. The role of the iliolumbar ligament in the lumbosacral junction. Spine 1990;15:1138-41.

9. Aihara T, Takahashi K, Yamagata M, Moriya H, Tamaki T. Biomechanical functions of the iliolumbar ligament in L5 spondylolysis. J Orthop Sci 2000;5:238-42.

10. Aihara T, Takahashi K, Yamagata M, Moriya H, Shimada Y. Does the iliolumbar ligament prevent anterior displacement of the fifth lumbar vertebra with defects of the pars? J Bone Joint Surg [Br] 2000;82-B:846-50

11. Fujiwara A, Tamai K, Kurihashi A, Yoshida H, Saotome K. Relationship between morphology of iliolumbar ligament and lower lumbar disc degeneration. J Spinal Disord 1999:12:348-52

12. Aihara T, Takahashi K, Ono Y, Moriya H. Does the morphology of the iliolumbar ligament affect lumbosacral disc degeneration? Spine 2002;27:1499-503.

13. Elster AD. Bertolotti's syndrome revisited: transitional vertebrae of the lumbar spine Spine 1989:14:1373-7.

14. Luoma K, Vehmas T, Raininko R, Luukkonen R, Riihimaki H. Lumbosacral transitional vertebra: relation to disc degeneration and low back pain. Spine 2004;29: 200-5.

15. Brown MF, Rockall AG, Hallam P, Hall-Craggs MA, Edgar MA. Transitional lumbo-sacral vertebrae: incidence of disc degeneration above and below [abstract] $J$ Bone Joint Surg [Br] 2000;82-B (Suppl II):180.

16. Castellvi AE, Goldstein LA, Chan DPK. Lumbosacral transitional vertebrae and their relationship with lumbar extradural defects. Spine 1984;9:493-5.

17. Vergauwen S, Parizel PM, Van Breusegem L, et al. Distribution and incidence of degenerative spine changes in patients with a lumbo-sacral transitional vertebra. Eur Spine J 1997:6:168-72.

18. Wigh RE, Anthony HE Jr. Transitional lumbosacral discs: probability of herniation Spine 1981:6:168-71

19. Otani K, Konno S, Kikuchi S. Lumbosacral transitional vertebrae and nerve-root symptoms. J Bone Joint Surg [Br] 2001;83-B:1137-40.

20. Fujiwara A, Lim TH, An HS, et al. The effect of disc degeneration and facet joint osteoarthritis on the segmental flexibility of the lumbar spine. Spine 2000;25: 3036-44.

21. Miller JAA, Schmatz C, Schultz AB. Lumbar disc degeneration: correlation with age, sex, and spine level in 600 autopsy specimens. Spine 1988;13:173-8.

22. Ghiselli G, Wang JC, Hsu WK, Dawson EG. L5-S1 segment survivorship and clinical outcome analysis after L4-L5 isolated fusion. Spine 2003;28:1275-80.

23. Videman T, Battie MC. Epidemiology of disc disease. In: Weinstein JN, Wiesel SW, eds. The lumbar spine. Second ed. Philadelphia: W.B. Saunders Co, 1996:16-27.

24. Battie MC, Videman T, Gibbons LE, et al. Determinants of lumbar disc degeneration: a study relating lifetime exposures and magnetic resonance imaging findings in identical twins. Spine 1995:20:2601-12.

25. Harford JM, McCullen GM, Harris R, Brown CC. The iliolumbar ligament. Spine 2000:25:1098-103.

26. Basadonna PT, Gasparini D, Rucco V. Iliolumbar ligament insertions: in vivo anatomic study. Spine 1996;21:2313-6.

27. Videman T, Sarna S, Battie MC, et al. The long-term effects of physical loading and exercise lifestyles on back-related symptoms, disability, and spinal pathology among men. Spine 1995;20:699-709.

28. Farfan HF, Cossette JW, Robertson GH, Wells RV, Kraus H. The effects of torsion on the lumbar intervertebral joints: the role of torsion in the production of disc degeneration. J Bone Joint Surg [Am] 1970;52-A:468-97.

29. Farfan HF. The torsional injury of the lumbar spine. Spine 1984;9:53. 